\title{
Characteristic Variability Time Scales of Long Gamma-Ray Bursts
}

\author{
Rongfeng Shen and Liming Song \\ Laboratory of Cosmic Rays and High Energy Astrophysics, Institute of \\ High Energy Physics, Chinese Academy of Sciences, Beijing, China
}

\begin{abstract}
We determine the characteristic variability time scales for 410 bright long GRBs by locating the maximums of their Power Density Spectra (PDSs) defined and calculated in the time domain. The averaged characteristic variability time scale decreases with peak fluxe. This is consistent with the time dilation effect expected by cosmological origin of GRBs. The occurrence distribution of the characteristic variability time scale shows bimodality, which might be interpreted as that the long GRB sample is composed of two sub-classes with different intrinsic characteristic variability time scales.
\end{abstract}

\section{Introduction}

Gamma-ray bursts (GRBs) have a complicated and irregular time profiles which vary drastically from one burst to another. The observed variability provides an intersting clue as to the nature of GRBs.

Li (2001) developed a new temporal analysis technique in the time domain without using Fourier transformation. We apply this technique and calculate the PDS in the time domain for 410 bright long bursts. The characteristic variability time scale of the burst can be obtained with this technique.

\section{PDS Calculation and Characteristic Variability Time Scale of The Burst}

The BATSE Concatenated 64-ms Data (ftp://cossc.gsfc.nasa.gov/pub/data/batse/ascii_data/64ms) summed over energy channel II and III $(50-300 \mathrm{keV})$ with the background subtracted are used. Bursts with $T_{90}>15 \mathrm{~s}$ are selected since short bursts are not suitable for calculating PDS. To avoid low $\mathrm{S} / \mathrm{N}$ ratios, we exclude dim bursts with peak count rates $<250$ counts per $64 \mathrm{~ms}$ bin. Within this two criterions a sample of 478 bursts are selected. Then we calculate PDS of each burst directly in the time domain.

Most of the bursts' PDSs show a "bump" shape. The bump-shape indicates that the variations taking place at a specified time scale dominate superiorly to variations at other time scales. Thus, we define the time scale at which the maximum of PDS locates as the time scale of typical variation in the burst's temporal profile, or, as the characteristic variability time scale $\left(\Delta t_{p}\right)$ of the burst. 

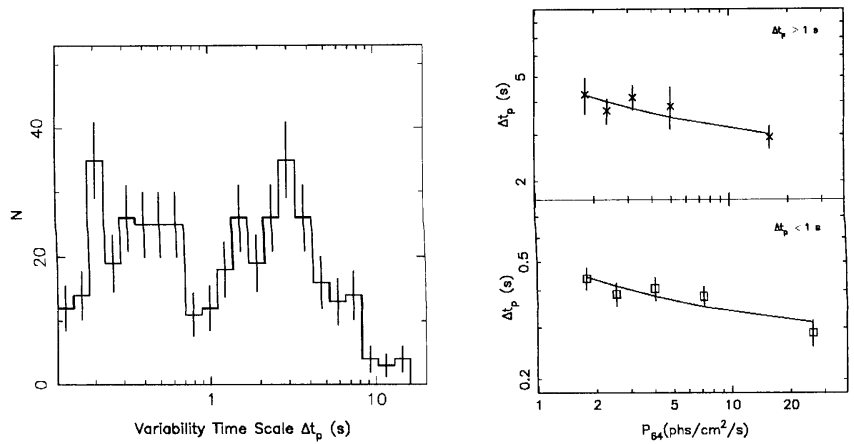

Figure 1. Left: Occurrence distribution of characteristic variability time scales for 410 bright long bursts. Right: $\Delta t_{p}$ vs. brightness distribution for the $\Delta t_{p}>1$ s group and the $\Delta t_{p}<1$ s group. Each bin includes equal number of samples. Solid lines are the best-fit model predictions.

\section{Distribution of $\Delta t_{p}$ and Time Dilation Test}

From the selected samples, the $\Delta t_{p}$ of 410 bursts are determined. Fig. 1(left) plots their histogram distribution. One can find it is a bimodal distribution with the demarcation at $\Delta t_{p} \sim 1 s$. The bursts can be divided naturally into two groups, namely fast variable group $\left(\Delta t_{p}<1 \mathrm{~s}\right)$ and slowly variable group $\left(\Delta t_{p}>1 \mathrm{~s}\right)$. Some researchers concentrate on reclassification of GRBs, and divided the canonical "long class" (Kouveliotou et. al. 1993) into two new subclasses (Mukherjee et al. 1998; Balastegui, et al. 2001). The bimodal distribution of $\Delta t_{p}$ is a new evidence that the long burst class comprises two sub-classes.

If a GRB occurs at a cosmological distance, every structure in the time profiles will be stretched by a factor $1+z$ due to the expanding universe, where $z$ is the red shift. One should observe that the dimmer bursts have larger $\Delta t_{p}$ than the brighter bursts do, assuming that bursts are "standard candles" and have the same intrinsic characteristic variability time scale.

We divide the bursts into $\Delta t_{p}>1 s$ group and $\Delta t_{p}<1 s$ group, and plot in Fig. 1(right) the distribution of mean of $\Delta t_{p}$ in 5 brightness bins for each group, where brightness is represented by $P_{64}$, the peak flux measured on $64 \mathrm{~ms}$ time scale. Both groups show that averaged $\Delta t_{p}$ decreases with the brightness, a trend consistent with the theoretical predications.

Acknowledgments. The authors thank S. N. Zhang, T. P. Li, W. F. Yu, F. J. Lu, and J. L. Qu for their useful discussions and valuable advices.

\section{References}

Balastegui, A., Ruiz-Lapuente, P., \& Canal, R. 2001, MNRAS, 328, 283

Kouveliotou, C., Meegan, C. A., Fishman, G. J. et al. 1993, ApJ, 413, L101

reference Li, T. P. 2001, CJAA, 1, 313

Mukherjee, S., Feigelson, E. D., Babu, G. J. et al. 1998, ApJ, 508, 314 